Onkologe 2016 $\cdot 22: 618-621$

DOI 10.1007/s00761-016-0060-2

(c) Springer-Verlag Berlin Heidelberg 2016

CrossMark

\author{
A. Glaus' $\cdot$ P. M. Schlag 2 \\ 'Tumor- und Brustzentrum ZeTuP, St. Gallen, Schweiz \\ ${ }^{2}$ Charité Comprehensive Cancer Center, Charité Campus Mitte, Berlin, Deutschland
}

\section{Onkologische Pflege im interdisziplinären Team}

\author{
Ein Paradigma moderner \\ patientenzentrierter Betreuung
}

Der angehende Wandel in Medizin und Pflege nimmt unaufhaltsam Einfluss auf die Gestaltung der onkologischen Betreuung. Die Zahl der Patienten steigt und der Bedarf an Betreuern ebenfalls. Die Schere zwischen Bedarf und Verfügbarkeit spezialisierter Pflegefachpersonen und Onkologen geht weiter auf. Dies spiegelt die gesellschaftliche Entwicklung und beschert große Herausforderungen. Aber auch die neue Bildungssystematik in der Pflege und interprofessionelle Entwicklungen in der Gesundheitsversorgung verändern den Alltag von Patienten, Ärzten und Pflegenden. Die Ressourcen sind zwar zunehmend limitiert, dennoch sind neue Formen von Versorgungsmodellen, Spezialisierung und neue Berufsbilder nicht nur ein Problem, sondern zugleich auch eine Chance.

Die onkologische Pflege ist ein Spezialgebiet, auf deren Anfänge sich in Europa noch gut zurückblicken lässt. Was in den 70er Jahren mit einigen Pionieren und neuen Medikamenten begann, ist heute ein Fach mit vielen Spezialitäten und Schnittstellen zwischen Disziplinen, wie z. B. Onkologie, Hämatologie, Immunologie, Chirurgie, Gynäkologie, Radiotherapie oder Pharmakotherapie.

Die Anwendung multimodaler Therapieformen verlangt nach großem Fachwissen und intensiver Kooperation. Zusammenarbeit und Kommunikation werden hier zu sehr zentralen Schwerpunkten. Dazu wollen Onkologiepflegende in dieser Ausgabe von Der Onkologe Stellung nehmen. Sie beschreiben aus ihrer Perspektive die Kommunikation zwi- schen Professionen im Betreuungsteam, Patienten und Betreuern, Angehörigen und dem Team oder zwischen und unter den Professionen im Behandlungsprozess. In aller Begrenztheit eines solchen Themenüberblicks geben sie punktuell Einblick in ihre Vorstellungen und Ansprüche der modernen Betreuung onkologischer Patienten, in der die Pflege hoch qualifizierte Rollen einnehmen kann und muss. Ganz dem Wesen der Pflege verpflichtet wurden dabei medizinische, aber auch psychologische, soziale und ethische Facetten und Pflegeaspekte ausgewählt.

\section{》) Multimodale Therapieformen erfordern großes Fachwissen}

Vorausgehend scheint es wichtig $\mathrm{zu}$ erwähnen, dass der oft kritisch beschriebene Wandel von Medizin und Pflege auch zu großen Fortschritten geführt hat So hat sich die Fachpflege in der Onkologie auch im deutschen Sprachraum auf verschiedenen Ebenen entwickelt. Nebst einem Abschluss in höherer Fachausbildung Onkologie haben sich viele Kollegen in den letzten Jahren auch für ein Studium in Pflege begeistern können, was ihnen gerade im interdisziplinären Team hilft, evidenzbasiertes Entscheiden und Handeln in die Praxis einzubringen und die Lehre und den Umgang mit Forschungsresultaten abzustützen. Das Reservoir an gut ausgebildeten und selbstbewussten Onkologie-Pflegefachkräften mit praxisorientierten, akademischen Abschlüssen, wie einem Master of
Advanced Studies (MAS) sowie einem Bachelor oder Master of Science (MSc), ist größer geworden. Die Zeit reift heran, in der professionelle Rollenteilungen zum Wohl der Patienten möglich werden. Das nährt die Hoffnung, dass bei Pflegenden die Zufriedenheit im Beruf wieder steigt, was nicht zuletzt die Zufriedenheit der Erkrankten in besonderem Maße prägen kann. Davon will dieses Heft zeugen.

Onkologische Patienten brauchen mehr als eine Krebstherapie. Die Integration der psychosozialen Betreuung in die pflegerische und medizinische Onkologie entspricht einem alten Postulat, welches leider auch heute noch $\mathrm{zu}$ selten systematisch in die Praxis umgesetzt ist. Unter diesem Blickwinkel beschäftigt sich der Beitrag von Glaus und Stolz mit den Belastungen, welche Krebskrankheiten und deren Therapien mit sich bringen. Es wird in Erinnerung gerufen, dass gerade bei onkologischen Erkrankungen die Seele mitleidet. So wird gefragt, wie es in der oft hektischen, klinischen Routine gelingen kann, Menschen mit einer hohen psychosozialen Belastung zu identifizieren, um entsprechende Hilfe zu vermitteln. Wann brauchen Patienten oder ihre Angehörigen mehr als die Betreuung im klinischen Onkologie-Team? Postuliert wird ein systematisches Screening-Modell, in dem Onkologie-Fachpflegende in ihrer Schlüsselposition Verantwortung im interdisziplinären Team übernehmen.

Ungenügende Kommunikation und mangelnde Zusammenarbeit in der Gesundheitsversorgung erschweren das 
Hier steht eine Anzeige.

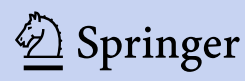


Erreichen guter Ergebnisse für Patienten und ihre Angehörigen erheblich, kosten $\mathrm{zu}$ viel Geld und senken die Patienten- und Mitarbeiterzufriedenheit. Eine Onkologie-Pflegeexpertin und ein Palliativmediziner widmen sich in diesem Heft der Frage „Wie kann interprofessionelle Zusammenarbeit besser gelingen?" Ein häufiger Grund für Dissonanzen im onkologischen Behandlungsteam sind aus klinischer Erfahrung oftmals Therapieentscheidungen, welche nicht von allen mitgetragen werden können. In der Palliativmedizin wird die Versorgung personenorientierter und verlässt den Diagnosefokus. Im Beitrag von Fliedner und Eychmüller wird vom Ausräumen von Vorurteilen, Wertschätzung der Berufskompetenz, kritischem Denken, aber auch von neuen Organisationsstrukturen nach Fachkompetenz und gemeinsamem Lernen gesprochen. Das Autorenteam plädiert für die Integration des Patienten in das Team und für eine gemeinsame Definition der Behandlungsziele. Vergleiche zwischen Team-Management-Modellen mit herkömmlicher Betreuung haben gezeigt, dass kooperative Modelle der Versorgung die besseren Resultate erzielen (vgl. auch [1]).

\section{》) Angehörige haben eine zentrale Rolle bei der Unterstüt- zung von Menschen mit Krebs}

Angehörige haben eine zentrale Rolle in der Betreuung, Begleitung und Unterstützung von Menschen mit Krebs. Angehörige von Krebskranken werden jedoch, wie Jähnke und Steudle in diesem Heft ausführen, nach wie vor kaum auf psychosoziale Unterstützungsmöglichkeiten angesprochen. Die Aussage, dass Angehörige als Deutschlands größter Pflegedienst bezeichnet werden, beschreibt die Dimension und Relevanz dieser Gruppe. Eine Sensibilisierung für die Bedürfnisse von Angehörigen innerhalb des interprofessionellen Betreuungsteams, Ansprechbarkeit und Kontinuität in der Betreuung sowie der wechselseitige Austausch sind wesentlich, um Angehörige gezielter zu unterstützen. Pflegefachpersonen können, Dank ihrer
Schlüsselposition, in der psychosozialen Begleitung und Unterstützung von Angehörigen einen wertvollen Beitrag leisten.

Die Grundzüge bei der Gestaltung eines multidisziplinären Symptommanagements werden im Beitrag von Titzer dargelegt. Es wird als sinnvoll betrachtet, sich dabei auf theoretische Modelle abzustützen, beispielhaft auf die Theorie von M. Dodd, welche diese als ein Gerüst mit Anhaltspunkten zur Implementierung eines umfassenden multiprofessionellen Symptommanagement vorsieht. Auch hier sind die Konzepte Kommunikation und Rollenaufteilung im Team von zentraler Bedeutung.

Die Notwendigkeit und Abstimmung einer inter- und transdisziplinären $\mathrm{Zu}$ sammenarbeit von ärztlichem, pflegerischem und medizintechnischem Personal wird praxisnah von Rau et al. am Beispiel der hyperthermen intraperitonealen Chemotherapie (HIPEC) beschrieben. Das interdisziplinäre AutorinnenTeam zeigt dabei die praktischen Aspekte der speziellen Zusammenarbeit zwischen Operationssaal, Intensivstation und Normalstation auf. Mit dieser neuen Behandlungsstrategie besteht ein zusätzliches Expositionsrisiko aller Mitglieder rund um den Operationssaal gegenüber Zytostatika. Damit werden die Sicherheitsaspekte und Sicherheitsrichtlinien den örtlichen sowie professionellen Abgrenzungen übergeordnet.

Abrundend zur Thematik des Hefts werden von Achatz ethische Betrachtungen zur onkologischen Pflege angestellt. Es wird darin ausgeführt, dass sich moralisches Urteilen und Handeln an den Grundprinzipien Autonomie, Fürsorge und Vertrauen orientieren muss, was eine kritische Reflexion unabdingbar macht. Alle im Gesundheitssystem Tätigen sowie die Institutionen sind angehalten, für ein Klima zu sorgen, das von gegenseitigem Vertrauen geprägt ist, um so die Autonomie der Patienten zu stärken und gleichzeitig eine fürsorgliche Pflegepraxis zu ermöglichen.

Somit repräsentieren die Beiträge in diesem Heft einen interessanten Ausschnitt aus dem großen Bündel der pflegerischen Arbeit mit onkologischen Patienten. Im Zusammenhang mit Dia- gnostik, Therapie, Pflege, Rehabilitation, Nachsorge und Palliation ergeben sich heute zahlreiche Aufgabenbereiche, in denen Pflege einen wichtigen Beitrag im interdisziplinären Team leisten kann und muss. Im Mittelpunkt steht dabei stets, dass die Sorge um den Menschen, das Caring, die entscheidende Grundlage in der Betreuung bleibt. Auch wenn Caring, historisch gesehen, am augenscheinlichsten bei der Pflege angesiedelt ist, ist es durchaus nicht nur der Pflege vorbehalten, sondern jeder Einzelne im interdisziplinären Team kann und muss auf diesem Fundament bauen. Davon profitieren dann alle, zuerst der Patient - aber auch das ganze Team.

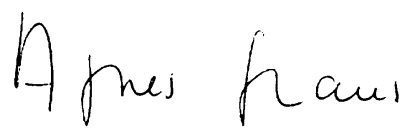

Dr. Agnes Glaus (PhD, MSc, RN)

für die Schriftleitung

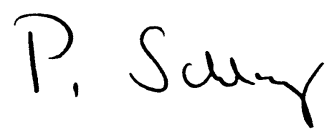

Prof. Dr. Peter M. Schlag

für die Herausgeber

\section{Korrespondenzadresse}

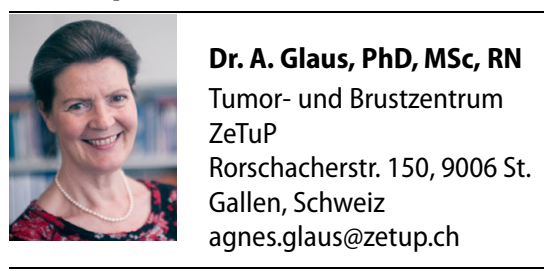

Interessenkonflikt. A. Glaus und P.M. Schlag geben an, dass kein Interessenkonflikt besteht.

\section{Literatur}

1. Martin J, Ummenhofer W, Manser T et al (2010) Interprofessional collaboration among nurses and physicians making a difference in patient outcome. Swiss Med Wkly 140:w13062 
Hier steht eine Anzeige.

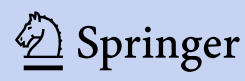

